- Fast track status reviewed early if not appropriate

- Coordination of care - having oversight of care at the end of life

- Having links to the hospice - work more effectively together

- Flexibility - challenge the standard ' $\mathrm{x} 2$ carers/x4 a day request' - refocus on patient-centred care

Typical 4-month stats (April - July 2019): Referrals - 296 and Care Hours - 6,331.75

Conclusion The primary objective of the service is to provide Individualised packages of care, increased/reduced according to need for patients in the last 6 weeks of life and those closest to them. Through this provision of home care, we are demonstrating that we can improve the assessment and management of symptoms.

\section{HOSPICE OUTREACH TEAM}

R Richardson, K Maw, D Willis. Severn Hospice

\subsection{6/spcare-2020-PCC.169}

Introduction The community nursing service was restructured to ensure that all adult people, regardless of their diagnosis, have choices and access to specialist palliative and end of life care; to strengthen the integration with our community colleagues; to improve anticipatory and advanced care planning; to reduce unplanned hospital admissions; and to ensure a timely response during episodes of palliative care crises.

Method To evidence a proposal for change an activity audit was completed, a triage system was tested, and views sought from GPs and community nursing leads. The team was changed and became comprised of two tiers of specialist nurse - Outreach Nurses and Outreach Practitioners who have extended skills of physical assessment and prescribing as well as overall responsibility for the wider caseload and the team. The service aimed to support GP's and District Nursing teams within cluster locations. The telephone triage facility was used to determine the appropriateness of referrals and the location for the first assessment, allowing care to be prioritised and the team to offer a responsive service to complex and urgent cases.

Results By managing complex, individual and changing information and supporting patients in choices around treatment and care we have demonstrating improvement in quality and experience along with an increase in referrals. Using and applying clinical knowledge to oversee and coordinate services, we can personalise the palliative pathway for individual patients and support the needs of their families. Additionally, we can act as the key accessible professional for the multidisciplinary team, undertaking proactive case management and using clinical acumen to reduce risk.

Typical 4-month stats (April - July 2019):

Referrals - 695

Home visits - 748

Triage calls - 1307

\section{OFF-LABEL PRESCRIBING RECOMMENDATIONS BY A HOSPITAL PALLIATIVE CARE TEAM (HPCT)}

Hannah Rose, Mark Banting. University Hospitals Southampton
Background It is well recognised that the off-label prescribing of medications is an essential part of practice in palliative care. 'Off-label' generally refers to a medicinal product that is used beyond the specification of its Marketing Authority. There are many reasons for the high incidence of such prescribing in palliative care including that the costly and timeintensive process of obtaining or expanding a licence can only be performed by a pharmaceutical company. Additionally, there is common use of subcutaneous medications, the majority of which are used off-label. While it is widely accepted as normal practice, there is a lack of standardisation regarding gaining informed consent from patients and its documentation.

Aim To gain information regarding the prevalence of off-label prescribing and address some of the issues that this presents.

Methods A retrospective study was conducted of the notes of patients referred to a HPCT over a 1 month period. All medications that were off-label (as determined by the Palliative Care Formulary, sixth edition and expert consensus) and recommended by the team were recorded including the drug, indication and route and whether by a HPCT consultant, doctor or Clinical Nurse Specialist (CNS).

Results 175 patients were referred. 541 drug recommendations were made, of which 266 (49\%) were for off-label medications. The most commonly recommended off-label medications included opioids for breathlessness and levomepromazine for nausea and vomiting. All members of the HPCT made recommendations at a similar frequency.

Discussion By determining the prevalence of off-label prescribing, we have an understanding of the volume, patterns of drugs, doses and routes and doctor versus CNS variability. This will guide education of the HPCT, improve teaching throughout our Trust and inform the development of Patient Information documentation.

\section{EVERY BREATH YOU TAKE : TRACHEOSTOMY VENTILATION IN CHILDREN'S PALLIATIVE CARE}

Elli Rushton, Christine Greenfield. Wessex Childrens Hospice Trust

10.1136/spcare-2020-PCC.171

Background Children's palliative care is changing. Medical and technological advances enable an increasing number of children with life limiting conditions to survive on long term ventilation (LTV) via tracheostomy. A specialist hospital identified that children spend months in hospital awaiting adaptations to accommodation, and establishment of a care package.

Aims

- Set up a unit for tracheostomy ventilated children within a Children's Hospice in partnership with the hospital. This unit would provide accommodation and nursing support for 3 children and families in preparation for discharge home.

- Facilitate care of child outside the hospital environment.

Methods October 2016-March 2017:

- Literature review

- Partnership working with respiratory team

- Agreed practice framework

March 2017-October 2017:

- Collaboration with specialist paediatric respiratory, and hospice palliative care teams 